LIBRO

James J. Heckman y Carmen Pagés (editores):

Law and Employment: Lessons from Latin America and the Caribbean

(University of Chicago Press, 2004, 573 páginas).

\title{
REFORMA LABORAL Y EMPLEO EN LATINOAMÉRICA*1
}

\author{
Sebastián Edwards
}

$\mathrm{E}_{\mathrm{n}}$ perdida”. Durante ese período el crecimiento per cápita fue negativo, el desempleo alcanzó niveles sin precedentes y la inflación se volvió extraordinariamente pertinaz. Estos deficientes resultados económicos fueron consecuencia de una serie de factores, entre ellos una excesiva sobrevaloración de los tipos de cambio reales, desequilibrios fiscales muy pronunciados, y un conjunto cada vez más complejo de regulaciones microeconómicas que derivaron en considerables distorsiones y en una marcada disminución de la productividad. Asimismo, cuando en 1982 México declaró que no podía pagar su deuda externa, los flujos de capital hacia Latinoamérica y el Caribe se suspendieron abruptamente, por lo que todos los países de la región se vieron obligados a experimentar procesos de estricto ajuste macroeconómico.

Sebastián Edwards. Cátedra Henry Ford II de Economía Internacional, Universidad de California, Los Angeles. Investigador asociado del National Bureau for Economic Research y coeditor del Journal of Development Economics. Entre 1993 y 1996 fue el Economista en Jefe para América Latina del Banco Mundial.

* La versión original de este artículo fue escrita en inglés. Traducción al castellano de Alberto Ide.

${ }^{1}$ Agradezco a Alejandra Edwards por sus comentarios de gran utilidad. 
Hacia fines de la década de 1980 ocurrieron en Latinoamérica tres importantes acontecimientos interrelacionados ${ }^{2}$ : En primer lugar, tras varios años de gobierno militar, la mayor parte de la región retornó a la vida democrática. En segundo lugar, se puso en marcha un programa en gran escala —el llamado Plan Brady — destinado a reestructurar la deuda externa. Y en tercer lugar, se implementaron progresivamente en todos los países una serie de reformas de amplio alcance orientadas al mercado. Si bien los aspectos específicos de estas reformas — entre ellos su oportunidad y profundidad - variaron de un país a otro, en la mayoría de los casos tuvieron algunos elementos esenciales en común, como por ejemplo la apertura del comercio internacional, la implementación de programas antiinflacionarios, la privatización de empresas estatales y la desregulación de los mercados.

A fines de los años ochenta y comienzos de los noventa, y como parte de esta campaña de modernización, una cantidad creciente de países latinoamericanos y caribeños empezaron a reformar su mercado laboral. En muchos de ellos la reforma del mercado laboral supuso una relajación de regulaciones aplicadas durante décadas; en otros, aumentó la seguridad del empleo y reforzó los derechos de los trabajadores. En el libro Law and Employment: Lessons from Latin America and the Caribbean, cuyos editores son James J. Heckman y Carmen Pagés (University of Chicago Press, 2004), se analiza la naturaleza de estas reformas y se investigan sus efectos en el empleo y en otros resultados del mercado laboral. La obra, en la que se sintetizan las conclusiones de un proyecto patrocinado por la American Bar Foundation y el Banco Interamericano de Desarrollo, se inicia con un extenso y extremadamente útil ensayo introductorio de los editores. A continuación sigue un capítulo sobre los salarios mínimos a cargo de William F. Maloney y Jairo Núñez Méndez, y capítulos por países dedicados a Perú (Jaime Saavedra y Máximo Torero), Colombia (Adriana D. Kugler, Mauricio Cárdenas y Raquel Bernal), Brasil (Ricardo Paes de Barros y Carlos Henrique Corseuil), Argentina (Guillermo Mondino y Sylvia Montoya; Hugo A. Hopenhayn), Chile (Claudio Montenegro y Carmen Pagés), Uruguay (Adriana Cassoni, Steven Allen y Gastón Labadie), y el Caribe (Andrew Dowes, Nlandu Mamingi y Rose-Marie Belle Antoine). El libro finaliza con un corto pero enjundioso ensayo sobre la demanda de trabajo en Latinoamérica y el Caribe, escrito por Daniel S. Hamermesh.

Las investigaciones discutidas en este volumen son particularmente importantes en el contexto chileno, donde después de años de debate, aún

${ }^{2}$ Latinoamérica y el Caribe conforman una región muy vasta, con 33 países distintos. Para una descripción detallada del proceso de reforma política y económica durante fines de los años ochenta y comienzos de los noventa, incluyendo informes por país, véase Edwards (1995). 
no se implementan nuevas reformas que modernicen la legislación laboral. Este tema, que ha sido considerado como "demasiado político" como para ser abordado en el último año de la administración Lagos, cobrará cada vez mayor importancia en los años venideros. Es difícil pensar que Chile pueda volver a crecer a tasas elevadas — por ejemplo, a un 7\% promedio anual, por tomar una cifra con la que muchos se han obsesionado - si no crea una economía altamente dinámica, y con una gran capacidad para adaptarse a las condiciones cambiantes de la globalización. Este libro permite despejar muchas de las dudas técnicas sobre los efectos de las regulaciones laborales en el funcionamiento de los mercados del trabajo.

La conclusión fundamental de este impresionante cúmulo de trabajo empírico es que las regulaciones del mercado laboral son importantes. Los editores resumen de la siguiente manera las conclusiones del proyecto:

La evidencia presentada en esta obra refuta una visión predominante según la cual las regulaciones del mercado laboral afectan sólo la distribución de los ingresos del trabajo e inciden sólo levemente en la eficiencia. Los resultados presentados en este volumen sugieren que los beneficios sociales obligatorios reducen el empleo y que las regulaciones sobre seguridad del empleo repercuten considerablemente en la distribución del empleo y en las tasas de rotación ... [L]as regulaciones sobre la seguridad del empleo fomentan la desigualdad entre grupos demográficos. (Página 2.)

\section{Los mercados laborales y el secuenciamiento de la reforma económica}

Durante mucho tiempo a los especialistas dedicados a estudiar los procesos de reforma económica les ha inquietado la secuencia en que se desregulan distintos mercados. La mayoría de los primeros análisis sobre este tema se concentraron en el secuenciamiento de las reformas al comercio internacional y a los mercados de capitales, y se preguntaban si las cuentas comerciales debían liberalizarse antes o después de que se levantaran las restricciones a la movilidad del capital internacional. Durante los años ochenta, sin embargo, varios autores se interesaron en el papel de la desregulación del mercado laboral en el proceso de reformas. Según la teoría comercial básica, el efecto de la liberalización del comercio es distinto en países con un mercado laboral completamente desregulado que en países con políticas intervencionistas en el mercado laboral. Tal vez el caso más sencillo sea el de un país con abundante fuerza de trabajo, un salario mínimo para toda la economía y un capital específico a un sector en el corto 
plazo. En ese caso una reforma comercial que reduzca (o elimine) los aranceles de importación derivará en desempleo (a corto plazo ${ }^{3}$ ). No obstante, si primero se suprime el salario mínimo — es decir, si el mercado laboral se desregula antes de que se implemente la reforma comercial—, no habrá desempleo. En tales circunstancias la fuerza de trabajo será redistribuida desde el sector anteriormente protegido (e intensivo en capital) hacia el sector exportador intensivo en trabajo; los salarios disminuirán respecto al numerario y aumentarán respecto a los productos importables. Con todo, este resultado no es muy sólido, y es altamente sensible a los supuestos formulados en cuanto a la cantidad de sectores de la economía, la naturaleza de la indexación salarial y la cobertura del salario mínimo. En definitiva, que el secuenciamiento de la reforma del mercado laboral dentro del proceso general de reformas revista o no importancia desde el punto de vista de la eficiencia es una cuestión empírica.

Muchos de los trabajos reunidos en esta obra se refieren al secuenciamiento de las reformas. Por ejemplo, en el capítulo introductorio Heckman y Pagés tratan de determinar si la reforma del mercado laboral en Latinoamérica y el Caribe tuvo lugar antes, al mismo tiempo, o después que la reforma comercial. También investigan la secuencia entre la reforma del mercado laboral y el retorno a la democracia tras un régimen militar. En esta indagación Heckman y Pagés distinguen entre dos tipos de reformas del mercado laboral: (a) la reforma laboral que disminuye el grado de protección jurídica de los trabajadores; y (b) la reforma laboral que aumenta el grado de protección del empleo de los trabajadores. En general descubren que no existe un claro patrón de secuenciamiento entre la reforma comercial y las reformas del mercado laboral (página 14). No obstante, Heckman y Pagés sí descubren que muchas reformas del mercado laboral tendían a ocurrir durante períodos de crecimiento económico negativo, y que muchas reformas que consolidaban los derechos de los trabajadores tenían lugar durante el período posterior al retorno a la democracia.

Si bien este tipo de análisis reviste importancia, pues aporta una percepción más clara de la economía política de las reformas, puede resultar engañoso. Un problema fundamental es la dificultad para determinar con precisión la fecha en que la reforma efectivamente ocurrió. Las reformas tienden a ser procesos dinámicos y progresivos, y no fenómenos discretos. ¿Debemos considerar que una reforma en particular se produjo el día en que

${ }^{3}$ En rigor, el resultado dependerá de si el salario mínimo se fija en función de los productos exportables o importables. Para que se obtengan los resultados analizados en el texto, el salario mínimo debería fijarse en función de los productos exportables, o de una canasta de productos donde los exportables tienen suficiente importancia. 
las autoridades la anunciaron, o el día en que se promulgó la nueva legislación? Y, ¿̇acaso cualquier enmienda en la legislación puede considerarse una reforma, o debemos concentrarnos en los grandes cambios? Heckman y Pagés tienen clara conciencia de este problema, y señalan que en su análisis "sólo se incluyen las grandes modificaciones de los códigos laborales u otras importantes intervenciones gubernamentales en el mercado laboral” (página 13, nota a pie de página 8). Aun así, el problema es que para investigar problemas de secuencia también tenemos que ser cuidadosos al establecer la fecha de otras reformas, incluidas, en particular, las reformas comerciales. Y en esta área Heckman y Pagés no son igualmente puntillosos. Por ejemplo, afirman incorrectamente que la reforma comercial chilena tuvo lugar a fines de 1984. Chile liberalizó el comercio entre 1976 y 1979; en 1983, y como una manera de afrontar la crisis de la deuda externa, Chile aplicó recargos temporales a los derechos de importación, pero el nivel general de protección siguió siendo muy bajo desde una perspectiva histórica y desde un punto de vista comparativo internacional. Además, de acuerdo con Heckman y Pagés la reforma comercial uruguaya se remonta a 1991. De hecho, la liberalización del comercio se implementó en Uruguay en 1978; luego de un breve retroceso durante las fases iniciales de la crisis de la deuda, la reforma comercial se intensificó en $1986^{4}$.

Con el objeto de profundizar nuestra percepción de los aspectos relativos al secuenciamiento de la reforma se requiere un análisis más minucioso del ordenamiento efectivo de las diversas reformas. Conjeturo que una vez concluido dicho análisis descubriremos que en la mayor parte de Latinoamérica y el Caribe la reforma laboral fue postergada y se emprendió hacia el final del proceso de reformas de modernización orientado al mercado.

\section{Medición de los costos de las regulaciones laborales}

Uno de los aportes más útiles de esta obra es el cálculo del costo de las regulaciones del mercado laboral para diversos países de Latinoamérica y el Caribe, lo mismo que para un grupo de naciones de la OCDE. Por ejemplo, en el ensayo introductorio, Heckman y Pagés determinan dos índices de costos de las regulaciones del mercado laboral: (a) los costos asociados a las cotizaciones previsionales (CP); y (b) los costos relativos a las leyes de seguridad del empleo (SE). Lo anterior se define de la siguiente manera:

${ }^{4}$ En estricto rigor, utilizan datos provenientes del BID (1996) para determinar las fechas de las reformas comerciales. Pero ello no significa que esas fechas sean correctas. De hecho, en varios casos resultan discutibles. 
(1) $C P_{j t}=\sum_{i=0}^{T} \beta^{i}\left(s s_{j, t+1}^{e}+s s_{j, t+1}^{w}\right)$,

donde $S S^{e}{ }_{j, t+1} \mathrm{y} \mathrm{SS}_{j, t+1}^{w}$ son los costos de los impuestos a la planilla que pagan el empleador y el empleado, expresados como porcentaje de los salarios, y $\beta$ es la tasa de descuento.

Por otra parte, el costo esperado de la seguridad del empleo se define como:

$$
S E_{j t}=\sum_{i=0}^{T} \beta^{i} \delta^{i-1}(1-\delta) b_{j, t+1}+\sum_{i=0}^{T} \beta^{i} \delta^{i-1}(1-\delta)\left[a_{j} y_{j, t+i} j c+\left(1-a_{j}\right) y_{j, t+i} u c\right] .
$$

$$
+\sum_{i=0}^{T} \beta^{i} c_{j, t+1}
$$

donde $\delta$ es la probabilidad de que un trabajador permanezca en su empleo durante un período determinado, $i$ es el período de permanencia en la firma $i$, T es el período máximo de permanencia (supuestamente 20 años), $b_{j, t+1}$ es la notificación previa de despido que se debe entregar a un trabajador con un período de permanencia de $i$ (medido en salarios mensuales); $a_{j}$ es la probabilidad de que las dificultades económicas que afronta la firma sean consideradas una causal de despido "justa”; y $c_{j, t+1}$ es el aporte (obligatorio) a la cuenta de ahorro de un trabajador. Al calcular (1) y (2) Heckman y Pagés suponen que la misma tasa de descuento (8\%) y tasa de despido (12\%) es aplicable a todos los países a lo largo del tiempo.

Sus resultados indican que los costos asociados a la seguridad del empleo son significativamente mayores en las naciones latinoamericanas que en los países de la OCDE. Por otra parte, los costos de las cotizaciones previsionales son mayores en los países avanzados que en Latinoamérica.

Descubrieron que a fines de los años noventa, en Latinoamérica y el Caribe, los costos de las indemnizaciones alcanzaban el nivel más alto en Perú, Colombia y Ecuador, y el más bajo en los países del Caribe. El costo promedio (esperado) de las indemnizaciones en Latinoamérica equivale a 2,46 salarios mensuales; en contraste, en los países avanzados de la OCDE esa cifra asciende a 0,8 salarios mensuales. Con respecto a las cotizaciones previsionales, los costos más altos se observan en Argentina y Uruguay, y los más bajos, una vez más, en el Caribe. A partir de sus cálculos, Heckman y Pagés concluyen que: 
Los países latinoamericanos y del Caribe soportan una carga más pesada de regulaciones que afectan los procesos de ajuste en el mercado laboral. Los países europeos soportan una carga más pesada de impuestos a la planilla que afectan la demanda de trabajo, pero no el ajuste laboral. (Página 31, sin destacar en el original.)

Un aspecto importante es el grado en que las cotizaciones previsionales son consideradas un impuesto por los trabajadores. Uno de los beneficios potenciales de las reformas a la seguridad social que crean cuentas individuales de ahorro previsional, como las reformas implementadas en muchos países latinoamericanos, es que incrementan el vínculo entre cotizaciones y (futuros) beneficios. En un sistema basado en cuentas individuales lo ideal sería que los empleados consideraran las cotizaciones en sus cuentas como una forma diferida de indemnización. En tal caso, el efecto de las cotizaciones previsionales en el mercado laboral sería significativamente distinto del observado en regímenes previsionales basados en el sistema de reparto, donde prácticamente no existe conexión entre cotizaciones y beneficios. En este tipo de sistema tradicional de reparto, las cotizaciones previsionales (o un alto porcentaje de ellas) suelen ser consideradas un impuesto; los beneficios son percibidos como un derecho.

En muchos de los estudios por país reunidos en este volumen se utilizan expresiones similares a (1) y (2) para determinar cómo evolucionan a través del tiempo los costos de las regulaciones del mercado laboral. Saavedra y Torero, por ejemplo, documentan con lujo de detalles la notable disminución en el costo de los programas de seguridad del empleo (indemnizaciones) en Perú entre 1991 y 1997. Según su índice, durante ese período los costos del programa de indemnizaciones para las empresas se redujeron a la mitad (en este cálculo se da por supuesta una constante probabilidad de despido). Cárdenas y Bernal proporcionan una estimación similar para Colombia, y demuestran que tras la reforma laboral de 1990 y la reforma previsional de 1993 los gastos no salariales en que incurrieron las empresas colombianas se incrementaron significativamente. Montenegro y Pagés elaboraron un índice amplio de los costos de la legislación de seguridad del empleo en Chile desde principios de la década de 1960. Muestran que en 1966 este costo aumentó de manera muy considerable hasta llegar al equivalente a 4 salarios mensuales promedio. Los costos disminuyeron drásticamente a menos de un salario promedio durante la primera etapa del régimen de Pinochet a mediados de los años setenta. Los costos subieron a 2,4 salarios durante la reforma laboral de 1981, y tras el retorno a la democracia en 1991 aumentaron aun más hasta llegar a 3 salarios. 


\section{Los efectos de las regulaciones laborales en los resultados del mercado laboral}

En esta obra los autores utilizan extensos conjuntos de microdatos para analizar los efectos de las regulaciones en (a) los niveles de empleo, y (b) los flujos de empleo. El primer efecto se relaciona con costos estáticos, mientras que el segundo tiene que ver con costos dinámicos o de transición. Se usaron dos enfoques metodológicos para examinar estos aspectos. En primer lugar se calcularon ecuaciones de demanda de trabajo para evaluar los efectos de las regulaciones en los niveles de empleo. En la medida en que las regulaciones aumenten el costo laboral, generarán un desplazamiento a lo largo de la curva de demanda y, por ende, una reducción del empleo. Si bien en la mayoría de los estudios por país se recurrió a funciones de demanda estática, en varios casos se hizo también un esfuerzo por incorporar aspectos relativos al ajuste. En segundo lugar, se calcularon tasas de rotación para comprender si las regulaciones afectaban la fluidez y la dinámica de las regulaciones laborales. Aun cuando en diversos capítulos los autores utilizan distintas técnicas específicas, en todas las colaboraciones se aplican cuidadosamente avanzados métodos econométricos y estadísticos.

\subsection{Demanda de trabajo}

La mayoría de los análisis sobre demanda de trabajo se basaron en los estudios de Bentolia y Saint Paul (1992) y de Burgess y Dolado (1989). Por ejemplo, Cárdenas y Bernal calcularon la siguiente ecuación para Colombia (esta especificación es representativa de la mayoría de los estudios reunidos en el volumen):

(3) $n_{t}=c+\alpha_{0} y_{t}+\alpha_{1} y_{i-1}+\beta_{0}\left(w_{t}+n w_{t}\right)+\beta_{1}\left(w_{t-1}+n w_{t-1}\right)+\gamma_{t} n_{t-1}+u_{t}$,

donde

(4) $\gamma_{t}=\gamma_{0}+\gamma_{1} R 1_{t}+\gamma_{2} R 2_{t}$

En la ecuación (3) $n$ es el empleo, $y$ es una predicción de la producción mediante una autorregresión rotatoria, $w$ es una predicción de salarios mediante una autorregresión rotatoria, y $n w$ son costos laborales no salariales. $\gamma$ mide el costo del ajuste y se supone que varía a través del tiempo y está determinado por la ecuación (4). En esta ecuación $R 1$ es un índice que mide el costo de las indemnizaciones, y $R 2$ mide el costo de los despidos. 
Estas ecuaciones han sido presentadas como series de tiempo, pero en la mayoría de los estudios por país también se utilizaron para las estimaciones datos de panel a nivel de empresas. Hay dos parámetros de interés en la estimación de este tipo de ecuaciones: la elasticidad precio de la demanda de trabajo con respecto a los costos laborales, y la velocidad de ajuste del mercado laboral a las perturbaciones. Como lo denota la ecuación (4), la velocidad de ajuste, que de alguna manera mide el grado de fluidez del mercado laboral, supuestamente depende del alcance de las regulaciones de seguridad del empleo, captado por los parámetros $R 1$ y $R 2$.

En la amplia mayoría de los estudios contenidos en este libro se descubrió que las elasticidades precio de la demanda de trabajo para producción constante eran significativamente negativas. Los valores estimados de estas elasticidades fluctuaban, para todos los trabajadores, entre $-0,17$ y $-0,69$; los valores para empleados de oficina oscilaban entre $-0,44$ y $-0,59$; y para los obreros, entre $-0,32$ y $-1,37$. Estos resultados indican claramente que en Latinoamérica y el Caribe las regulaciones del mercado laboral que aumentan los costos laborales repercuten negativamente en el nivel de empleo. Es más, en términos cuantitativos este efecto es al parecer muy importante: la elasticidad promedio estimada para todos los trabajadores asciende a $-0,28$, lo cual indica que la reforma del mercado laboral que reduce los costos laborales en un 10\% generará un aumento de 2,8\% en el nivel de empleo ${ }^{5}$.

Los estudios reunidos en este volumen sugieren que la velocidad de ajuste de los mercados laborales latinoamericanos ha sido más bien lento; a decir verdad, más lento que en las naciones avanzadas. Aquellos autores que procuraron determinar si la velocidad de ajuste había variado como reacción frente a cambios en las regulaciones, obtuvieron resultados contradictorios: mientras Saavedra y Torero descubrieron que al aumentarse las regulaciones disminuía considerablemente la velocidad de ajuste en Perú, Cárdenas y Bernal, lo mismo que Paes de Barros y Conseuil, concluyeron que en Colombia y Brasil las reformas no incidían significativamente en aquélla.

\subsection{Seguridad del empleo, empleo y rotación}

Desde un punto de vista teórico, los efectos de la legislación de seguridad del empleo en el nivel de empleo son ambiguos. Lo anterior se

${ }^{5}$ Dentro de este marco no es posible saber cuál es el efecto de las regulaciones en la tasa de desempleo. Para abordar este problema necesitaríamos un marco claramente definido que nos permita analizar las decisiones sobre la oferta de trabajo. 
debe a que este tipo de legislación produce dos efectos compensatorios: por una parte, se desalienta la contratación; por otra, los despidos tienden a disminuir. Aun así, la mayor parte de los modelos teóricos sugiere que la legislación de seguridad del empleo reduce sin lugar a dudas la tasa de rotación laboral. Asimismo, según algunos modelos recientes —entre ellos los modelos propuestos por Kugler, Pagés y Montenegro en este volumen-, la legislación de seguridad del empleo afecta la composición del empleo, reduciendo el nivel de empleo en el sector formal (o sector que acata las regulaciones) e incrementándolo en el sector informal.

La mayoría de los estudios por país comprendidos en este volumen recurrieron a cierta forma de estimación de diferencias-en-las-diferencias para analizar el efecto de las modificaciones a la legislación en las tasas de rotación y otros resultados del mercado laboral. Uno de los desafíos de este tipo de análisis consiste en definir el "grupo de control" de trabajadores que no son afectados por los cambios en la regulación (o tratamiento). El grueso de los estudios utiliza alguna definición del sector "informal” como grupo de control. Por ejemplo, en su estudio sobre Perú, Saavedra y Torero definen los sectores formal e informal en base a criterios jurídicos: un individuo se incluye dentro del sector formal si durante ese año perteneció a un sindicato, suscribió un plan de pensiones o un seguro de salud. En su capítulo dedicado a Colombia, Kugler define como trabajadores del sector formal a aquellos que imponen en la seguridad social. En el estudio acerca de Brasil realizado por Paes de Barros y Corseuil, los trabajadores informales son definidos como aquellos que carecen de un contrato laboral ${ }^{6}$. Aunque en principio estas definiciones parecen razonables, adolecen de algunas limitaciones, incluido el hecho de que los criterios utilizados para definir el grupo de control —informalidad laboral — no son invariantes frente al "tratamiento" (esto es, frente al cambio en las regulaciones). Éste no es, sin embargo, un tema fácil de abordar, y es un problema que se presenta en la mayoría de los estudios de este tipo.

Los resultados presentados en este volumen indican que las regulaciones más estrictas de protección del empleo tienden a reducir la rotación del trabajo, como también el grado de flexibilidad de los mercados laborales. Además, Montenegro y Pagés descubrieron que la legislación chilena de seguridad del empleo repercutía negativamente en las mujeres y en los trabajadores menos calificados y, por ende, tendía a hacer más desigual la distribución del ingreso.

${ }^{6}$ En Brasil todos los trabajadores cuentan con un documento (la carteira de trabalho) donde están registrados los principales aspectos de los contratos de trabajo (actual y anteriores). La existencia de este documento facilita la separación en grupos de trabajadores con y sin contrato. 


\subsection{Comparaciones internacionales}

En el capítulo introductorio, Heckman y Pagés reproducen los resultados de un estudio comparativo sobre las experiencias de países latinoamericanos y de la OCDE con regulaciones del mercado laboral ${ }^{7}$. Utilizan un panel desequilibrado para el período 1983-1999, con 417 observaciones que cubren 23 países avanzados de la OCDE y 15 naciones latinoamericanas. Buscan comprender la manera en que sus mediciones de las regulaciones del mercado laboral — tal como se definen en las ecuaciones (1) y (2) más arriba - afectan las tasas (agregadas) de empleo y desempleo en estos dos grupos de países. Ellos calculan una serie de modelos de efectos fijos para la muestra completa, al igual que para las muestras aisladas de países latinoamericanos y de la OCDE. Además de diversos índices de los costos de las regulaciones, las regresiones incluyen el PGB per cápita, el crecimiento real, como también diversos controles demográficos.

Heckman y Pagés descubrieron que el aumento de las imposiciones previsionales ha derivado en una reducción del nivel de empleo y en un aumento de la tasa de desempleo. Este resultado es válido para toda la muestra, al igual que para la muestra aislada de países latinoamericanos y de la OCDE. También se encontraron con que la "indemnización por antigüedad” había tenido un efecto positivo en el empleo, mientras que el pago de desahucios había incidido negativamente en el nivel de empleo en los países de la OCDE. Según estas estimaciones, todas las variables que miden los costos de las regulaciones tienen coeficientes positivos en la ecuación de desempleo para Latinoamérica; con todo, los coeficientes se calculan de manera imprecisa y no son significativos en niveles convencionales. El resultado más importante que se desprende de este análisis es que cuando todos los componentes de los costos de las regulaciones se combinan en un solo indicador, su coeficiente es considerablemente negativo en la ecuación de empleo, y considerablemente positivo en la regresión de desempleo.

\section{Salarios mínimos y mercados laborales}

El papel de los salarios mínimos ha sido objeto de debates por largo tiempo entre los responsables de formular políticas en Latinoamérica. Durante los últimos años esta discusión ha adquirido un carácter más general, por cuanto una serie de detractores de las reformas orientadas al mercado

${ }^{7}$ Esta es una versión ampliada de un trabajo anterior de los autores, Heckman y Pagés (2000). 
sostienen que ellas se han traducido en un empeoramiento de las condiciones sociales. De acuerdo con estos críticos, el alza en los salarios mínimos ayudaría a reducir la pobreza y contribuiría a mejorar las condiciones sociales de la región. Sin embargo, esta controversia en torno a las políticas se ha caracterizado por una falta de evidencias empíricas convincentes sobre los efectos de los salarios mínimos en los mercados laborales de la región.

En el capítulo a cargo de Maloney y Núñez Méndez se presenta uno de los pocos estudios empíricos exhaustivos acerca del impacto de la legislación sobre el salario mínimo en un grupo de países latinoamericanos (Argentina, Bolivia, Brasil, Chile, Colombia, Honduras, México y Uruguay). Los autores utilizan gráficos de densidad kernel para determinar si hacia fines de los años noventa los salarios mínimos eran obligatorios en los países de su muestra. Sus resultados pueden resumirse de la siguiente manera: al parecer la legislación sobre el salario mínimo fue obligatoria en todos los países, y produjo un efecto particularmente importante en los mercados laborales de Colombia, Honduras, Brasil y Chile. Más aun, existen evidencias de que el salario mínimo sirve como punto de referencia para todos los sectores de la economía, incluidos aquellos que no están legalmente obligados a aplicarlo (vale decir, el sector "informal”). Este hallazgo resulta de particular importancia por cuanto sugiere que los modelos de mercados laborales duales que suponen una flexibilidad salarial en el sector informal (o no cubierto) tal vez no sean particularmente aplicables a los casos de la región latinoamericana.

Maloney y Núñez Méndez usan además datos detallados de paneles rotatorios para Colombia, a fin de determinar cómo las alzas en el salario mínimo repercuten en algunas de las variables más importantes del mercado laboral ${ }^{8}$. Descubrieron que las alzas en el salario mínimo afectan la distribución de los salarios en el vecindario del propio salario mínimo, lo que se traduce en salarios más altos tanto para quienes ganan menos que el mínimo como para quienes reciben más que el mínimo (hasta cuatro veces el salario mínimo). Este resultado contrasta con el caso de las naciones avanzadas, donde los cambios en el salario mínimo afectan a un segmento mucho más reducido de la distribución salarial. Maloney y Núñez Méndez también descubrieron que el alza de los salarios mínimos incidía negativamente en el empleo. El estudio dedicado a Chile, realizado por Montenegro y Pagés, sugiere que el aumento del salario mínimo tiene un efecto negativo en la probabilidad de empleo de los trabajadores menos calificados y de las mujeres.

${ }^{8}$ Desde 1997 Colombia ha creado un panel rotatorio en el que se entrevista al mismo grupo familiar en dos encuestas consecutivas. 


\section{Aspectos adicionales, enfoques alternativos e investigaciones futuras}

Los estudios reunidos en este volumen proporcionan una impresionante cantidad de evidencias sobre el funcionamiento de los mercados laborales en Latinoamérica y en el Caribe. En particular, ellos muestran convincentemente que las regulaciones del mercado laboral tienen repercusiones: las regulaciones que aumentan el costo laboral (principalmente las cotizaciones previsionales) tienden a reducir el nivel de empleo; en tanto que las regulaciones que aumentan la seguridad del empleo tienden a reducir la fluidez de los mercados laborales y a reducir la velocidad del proceso de ajuste del mercado laboral.

Así y todo, existen diversos aspectos relacionados con el mercado laboral que aún no son suficientemente comprendidos. Las investigaciones posteriores en esta área deberían concentrarse en los efectos de la globalización, en las consecuencias de otras regulaciones, y en la interacción de la regulación del mercado laboral con la formación del capital humano. En lo que resta de esta sección me referiré a algunos temas para futuras investigaciones, y me ocuparé de algunos problemas relativos a la metodología y a las mediciones planteados en este libro editado por Heckman y Pagés.

Durante los últimos años la mayor parte de los países latinoamericanos ha procurado llegar a acuerdos de libre comercio con las naciones

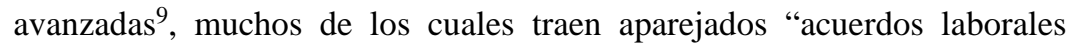
laterales", en los que las naciones latinoamericanas se comprometen a observar ciertas prácticas laborales. Un análisis de la manera en que las regulaciones incorporadas en estos acuerdos laterales afectan el equilibrio del mercado del trabajo ayudaría a determinar los verdaderos costos y beneficios de estas negociaciones sobre libre comercio. En este mismo sentido, el análisis de otras regulaciones laborales, como las contenidas en diversas convenciones de la OIT — por ejemplo, las que conciernen al trabajo infantil—, sería de gran utilidad para formarse una idea más clara acerca de cómo el aumento de la globalización podría afectar a los mercados emergentes en general, y a los países latinoamericanos en particular.

La mayoría de los estudios que conforman este libro han recurrido al cálculo de ecuaciones de demanda de trabajo-producto constante. Así y todo, en un mundo cada vez más globalizado resulta fundamental comprender de qué manera los cambios en la producción derivados de modificacio-

${ }^{9}$ Por ejemplo, al momento de redactarse este trabajo, dos países —Chile y México- ya han suscrito tratados de libre comercio con Estados Unidos, y un acuerdo similar entre Estados Unidos y naciones centroamericanas se encuentra a la espera de su ratificación por los congresos nacionales. 
nes en los términos de intercambio internacionales, o de variaciones en las propias regulaciones laborales, afectan los resultados del mercado laboral. En este tipo de trabajo sería natural incorporar en el futuro la reacción de la producción en el análisis de las consecuencias de las regulaciones del mercado laboral.

Uno de los aportes más importantes de esta obra es la elaboración de índices que miden los costos asociados a distintas regulaciones del mercado laboral, incluidos el pago de desahucios, las imposiciones previsionales, la notificación anticipada y la indemnización por antigüedad (véanse las ecuaciones (1) y (2) más arriba). A decir verdad, la construcción de estos índices cumple un papel decisivo en la estrategia de investigación que se sigue en esta obra: por una parte, se utilizan en las regresiones que determinan el efecto de las regulaciones en el empleo; por otra, estos índices ayudan a establecer la cronología de las reformas, de modo que resultan fundamentales para los análisis de diferencias-en-las-diferencias que comparan los resultados de los mercados laborales antes y después de las reformas a que se los somete. Sin embargo, como lo admiten los propios Heckman y Pagés, existen numerosas dificultades para elaborar estos índices. Por ejemplo, en el ensayo introductorio estos autores señalan:

En [nuestra] medición del costo de las regulaciones se omiten algunos componentes importantes del costo laboral. Por ejemplo, los costos de acatar ciertas leyes son difíciles de cuantificar y se omiten ... [E]sta medición no incluye el costo de regular la duración de la semana laboral estándar y de las horas extraordinarias. No incluye el costo de cumplir con las leyes sobre salario mínimo u otros niveles mínimos de ingreso. No incluimos las regulaciones de contratos de trabajo temporales. (Página 24.)

Estas dificultades para medir efectivamente los costos totales de las regulaciones sugieren que podría resultar útil aplicar un enfoque complementario como una manera de verificar la solidez de los resultados. Una auspiciosa vía de indagación consistiría en calcular ecuaciones de forma reducida para el comportamiento del mercado laboral, y tratar de establecer si es posible detectar la presencia de fallas estructurales en los datos. De hecho, si las reformas del mercado laboral incidieran en los resultados laborales, observaríamos cambios estructurales en los parámetros de las ecuaciones laborales clave ${ }^{10}$. En un reciente trabajo sobre la experiencia chilena con la reforma laboral, Edwards y Edwards (2000) adoptaron este enfoque y calcularon una ecuación del siguiente tipo sobre la dinámica del desempleo:

${ }^{10}$ Véase, por ejemplo, Burguess y Dolado (1989). Véase también la ecuación (4) más arriba. 
(5) $u_{t}=\alpha_{t}+\beta_{t} u_{t-1}+\gamma\left(g^{*}-g\right)+\varepsilon_{t}$.

donde $u_{t}$ es la tasa de desempleo en el período $t ; g^{*}$ es la tendencia a largo plazo del crecimiento real del PGB per cápita; $g$ es la tasa efectiva de crecimiento del PGB per cápita, y $\varepsilon$ es un error de media cero. El coeficiente $\beta(0 \leq \beta \leq 1)$ mide el grado de persistencia del desempleo y se supone que varía a través del tiempo; $\alpha$ se relaciona con el desempleo "de equilibrio" a largo plazo (esto es, con la tasa natural de desempleo), y también se permite que varíe a lo largo del tiempo ${ }^{11}$. Si una reforma del mercado laboral redujera los costos de las regulaciones, cabría esperar que el grado de persistencia del desempleo (es decir, el coeficiente $\beta$ ) disminuyera considerablemente en una época cercana a la fecha de la reforma ${ }^{12}$. Asimismo, a partir del cálculo de esta ecuación es posible determinar si la tasa "natural" de desempleo experimentó un cambio (estadísticamente significativo) en una época próxima a la fecha de las reformas. Edwards y Edwards (2000) utilizaron datos sobre Chile para el período 1960-1999 con el objeto de calcular la ecuación (5) aplicando la técnica del filtro de Kalman con coeficientes que varían a través del tiempo. Sus resultados sugieren que el grado de persistencia del desempleo experimentó un apreciable descenso en 1982, un año después de la reforma. Estas estimaciones indican además que entre 1988 y 1989 se observó una disminución estadísticamente importante en la tasa natural de desempleo de Chile.

\section{Comentarios finales}

Heckman y Pagés han recopilado un conjunto sumamente impresionante y útil de estudios sobre los mercados laborales y sus regulaciones en Latinoamérica. En los años venideros este volumen se transformará en la principal obra de consulta sobre la materia. Los futuros investigadores tendrán como punto de partida las colaboraciones reunidas aquí, y la calidad de los trabajos que se emprendan de ahora en adelante será evaluada en función de los altos estándares impuestos por este libro.

En el último ensayo del volumen Hamermesh resume con acierto y concisión los resultados de este conjunto de estudios. Parece apropiado finalizar esta recensión citando directamente a Hamermesh:

En conjunto, las evidencias sobre Latinoamérica [presentadas en este libro] deberían aumentar considerablemente el grado de seguridad

${ }^{11}$ La tasa de desempleo "natural" a largo plazo está dada por: $a /(1-b)$.

12 Blanchard y Summers (1986) analizan la manera en que las regulaciones del mercado laboral inciden en el grado de persistencia del desempleo. 
con que los economistas y asesores políticos hacen hincapié en los costos económicos a largo plazo de las llamadas políticas de protección del empleo ... Deberían subrayar la irrelevancia intrínseca de una profusión de modelos teóricos matemáticamente ingeniosos, basados esencialmente en argumentos acerca de las imperfecciones del mercado, que sostienen que esas políticas en realidad pueden aumentar el nivel de empleo ... También deberían sembrar en nosotros una profunda duda frente a los resultados empíricos arrojados por las comparaciones entre países que sostienen que dichas políticas no repercuten en los niveles de empleo. (Página 557.)

\section{REFERENCIAS}

Banco Interamericano de Desarrollo (BID): Making Social Services Work. Washington, D.C.: BID, 1996.

Bentolia, Samuel y Gilles Saint Paul: "The Macroeconomic Impact of Flexible Labor contracts with an Application to Spain”. En European Economic Review, 36:1013-53 (1992).

Blanchard, Olivier y Lawrence Summers: "Hysteresis and the European Unemployment Problem”. En NBER Macroeconomics Annual, 1986.

Burgess, Simon y Juan Dolado: "Intertemporal Rules with Variable Speeds of Adjustment: An Application to U.K. Manufacturing Employment”. En The Economic Journal, 99:347-65 (1989).

Edwards, Sebastián: Crisis and Reform in Latin America: From Despair to Hope. Oxford y Nueva York: Oxford University Press, 1995.

Edwards, Sebastián y Alejandra Cox Edwards: "Economic Reforms and Labour Markets: Policy Issues and Lessons from Chile”. En Economic Policy, 30: 182-229 (2000).

Heckman, James J. y Carmen Pagés: "The Costs of Job Security Regulation: Evidence from Latin American Labor Markets”. En Economia, 1: 109-54 (2000).

Heckman, James J. y Carmen Pagés: Law and Employment: Lessons from Latin America and the Caribbean. Chicago: University of Chicago Press, 2004. 